\title{
PARÂMETROS DE RUGOSIDADE AERODINÂMICA SOBRE VEGETAÇÃO ESPARSA EM REGIÃO SEMI-ÁRIDA ${ }^{1}$
}

\author{
GUSTAVO BASTOS LYRA ${ }^{2}$ e ANTONIO ROBERTO PEREIRA ${ }^{3}$
}

\author{
${ }^{2}$ Universidade Federal de Alagoas, Instituto de Ciências Atmosféricas. \\ Cidade Universitária, km 14, Rodovia BR 104, Tabuleiro dos Martins, Maceió, AL, CEP:57072-970. \\ E-mail: gblyra@gmail.com \\ ${ }^{3}$ Universidade de São Paulo, Escola Superior de Agricultura "Luiz de Queiroz", Dep. de Ciências Exatas. \\ Av. Pádua Dias, 11, Piracicaba, SP, Caixa Postal 9, CEP 13418-900. \\ E-mail: arpereir@esalq.usp.br
}

Recebido Novembro 2005 - Aceito Setembro 2006

\begin{abstract}
RESUMO
Utilizando observações micrometeorológicas e da estrutura física da vegetação do experimento HAPEXSahel foi possível avaliar alguns métodos para determinar o comprimento de rugosidade $\left(\mathrm{z}_{0}\right)$ e o deslocamento do plano zero (d). Propõe-se também uma alternativa para estimar esses parâmetros em escoamentos atmosféricos sobre vegetação esparsa em região semi-árida. O método proposto combinou o perfil logarítmico do vento com $\mathrm{z}_{0}=\lambda(\mathrm{h}-\mathrm{d})$, em que, h é a altura da vegetação e $\lambda$ depende de sua estrutura física. Valores de $\lambda$ foram estimados em função da estrutura física da vegetação ou obtido na literatura. A altura média da vegetação era $2,06( \pm 0,47) \mathrm{m}$. O perfil do vento foi caracterizado por medidas a quatro níveis acima da superfície $\left(3,0 ; 4,1 ; 5,3\right.$ e 8,5 m), com a velocidade de fricção $\left(\mathrm{u}_{*}\right)$ determinada por correlações dos turbilhões a $9 \mathrm{~m}$. O método proposto estimou adequadamente a rugosidade aerodinâmica da superfície $\operatorname{com} \lambda=0,188$ e 0,190, determinados pela estrutura da vegetação local, o que não ocorreu para $\lambda=0,166$, da literatura. Os parâmetros de rugosidade determinados pelos métodos que utilizam u* na solução da lei logarítmica do vento descreveram melhor a rugosidade da superfície. Nos períodos de observações a rugosidade da área foi descrita satisfatoriamente por $\mathrm{d}=0,95 \mathrm{~m}=0,46 \mathrm{~h}$ e $\mathrm{z}_{0}=0,204 \mathrm{~m}=0,1 \mathrm{~h}$.

Palavras-chave: HAPEX-Sahel; deslocamento do plano zero; comprimento de rugosidade; transporte de momentum.
\end{abstract}

\begin{abstract}
AERODYNAMIC ROUGHNESS PARAMETERS OVER SPARSE VEGETATION IN A SEMI-ARID REGION.

Using micrometeorological observations and the physical structure of the vegetation during the HAPEXSahel experiment it was possible to evaluate some methods to determine the roughness length $\left(\mathrm{z}_{0}\right)$ and the zero-plane displacement (d). It is also proposed an alternative to estimate such parameters for the atmospheric flow over sparse vegetation in a semi-arid region. It combines the wind logarithmic profile with $\mathrm{z}_{0}=\lambda(\mathrm{h}-\mathrm{d})$, where, $\mathrm{h}$ is the vegetation height, and $\lambda$ is a coefficient determined by the physical structure of the vegetation. Here, $\lambda$ values were determined for the site and also taken from the literature. The average vegetation height in the site was $2.06( \pm 0.47) \mathrm{m}$. The wind logarithmic profiles were defined by measurements at four level above the surface (at 3.0, 4.1, 5.3 and $8.5 \mathrm{~m}$ ), and the friction velocity $\left(\mathrm{u}_{*}\right)$ determined by eddy correlations at $9 \mathrm{~m}$. The proposed method estimated well the surface roughness with $\lambda=0.188$ and 0.190 , both determined by the structure of the vegetation in the site, but failed with $\lambda=0.166$, the average value of literature. The roughness parameters determined by methods that used $\mathrm{u}_{*}$ in the solution of the wind logarithmic law better described the surface roughness. The aerodynamic roughness of the site was described well by $\mathrm{d}=0.95 \mathrm{~m}=0.46 \mathrm{~h}$ and $\mathrm{z}_{0}=0.204 \mathrm{~m}=0.1 \mathrm{~h}$.
\end{abstract}

Keywords: HAPEX-Sahel; zero-plane displacement; roughness length; momentum transfer.

\footnotetext{
1 Parte da Tese apresentada pelo primeiro autor a Escola Superior de Agricultura "Luiz de Queiroz”, Universidade de São Paulo - ESALQ/USP, para obtenção do título de Doutor.
} 


\section{INTRODUÇÃO}

Na parametrização dos processos de transporte turbulento de momentum, calor e massa na camada de ar imediatamente acima de vegetações é fundamental conhecer o valor da rugosidade aerodinâmica da superfície. A rugosidade é descrita pelo comprimento de rugosidade $\left(\mathrm{z}_{0}\right)$ e deslocamento do plano zero (d). Estes parâmetros são essenciais em modelos baseados no método aerodinâmico (fluxo-gradiente) e em correções de medidas obtidas por correlação de vórtices turbulentos (DE BRUIN \& VERHOEF, 1997; TAKAGI et al., 2003). A teoria da similaridade de Monin \& Obukhov (1954) utiliza d para expressar os coeficientes de transporte turbulento e para caracterizar as condições de estabilidade atmosférica (MONTEITH \& UNSWORTH, 1990).

Algebricamente, $\mathrm{z}_{0}$ e d são apenas constantes de integração do perfil vertical da velocidade horizontal do vento (eq. 1) acima de uma superfície com obstáculos de porte alto e sob condições adiabáticas. Assim, $\mathrm{z}_{0}$ é a altura na qual a velocidade horizontal do vento tende a zero, ou seja, é o limite inferior de validade do perfil médio; e d é um escalar empírico para compensar o deslocamento vertical da dissipação de momentum pela superfície com obstáculos, isto é:

$$
\mathrm{U}_{\mathrm{z}}=\left(\frac{\mathrm{u}_{*}}{\mathrm{k}}\right) \ln \left(\frac{\mathrm{z}-\mathrm{d}}{\mathrm{z}_{0}}\right) \mathrm{z}>\mathrm{z}_{*}
$$

em que, $U_{z}$ é a velocidade horizontal média do vento à altura $z$; k é a constante de von Kármán; $u_{*}$ é a velocidade de fricção e $Z_{*}$ * é o limite superior da subcamada atmosférica rugosa (SCR).

São diversos os métodos para determinação de $\mathrm{z}_{0} \mathrm{e} \mathrm{d}$ baseados na eq. (1). Esses métodos requerem observações de $\mathrm{U}_{\mathrm{z}}$ na subcamada inercial $(\mathrm{z}>\mathrm{z} *)$ da camada limite de fluxo constante ou de equilíbrio (GARRATT, 1980; MONTEITH \& UNSWORTH, 1990) e bordadura (fetch) com extensão entre 50 e 100 vezes a maior altura de medida da velocidade do vento (WIERINGA, 1993; PEREIRA, 2002). Contudo, tais condições são difíceis de serem satisfeitas sobre vegetação esparsa e de porte alto, como no caso de arbustos e florestas (MOLION \& MOORE, 1983; DE BRUIN \& MOORE, 1985; LLOYD et al., 1992).

No método gráfico ou analítico convencional (PEREIRA, 2002), $z_{0}$ e d são determinados pelo ajuste da eq. (1) com valores de $\mathrm{U}_{\mathrm{z}}$ medidos, no mínimo, em quatro alturas acima da vegetação (WIERINGA, 1993). O ajuste é feito por tentativa e erro buscando o melhor ajuste da eq. (1). Freqüentemente o método convencional produz resultados incoerentes, principalmente para vegetação esparsa e de porte alto, em condições de bordadura limitada e de medidas no interior da subcamada rugosa (RAUPACH et al., 1980). A abordagem convencional tem a desvantagem de estimar três parâmetros $\left(\mathrm{d}, \mathrm{z}_{0}\right.$ e $\left.\mathrm{u}_{*}\right)$ com apenas uma equação. Estes parâmetros são inter-relacionados e obtidos por processo iterativo, não resultando necessariamente em solução física adequada (JACOBS \& van BOXEL, 1988; SCHAUDT, 1998). Devido a isso, o método torna-se subjetivo e sensível a erros (MOLION \& MOORE, 1983). Para minimizar esse problema, alguns autores (JACOBS \& van BOXEL, 1988; LLOYD et al., 1992; TAKAGI et al., 2003) combinaram a eq. (1) com $u_{*}$ determinado por correlações de vórtices turbulentos, o que resultou em estimativas mais satisfatórias. A determinação independente de $\mathrm{u} *$ exige sensores sofisticados, sistema de aquisição de dados e software específico para cálculo das covariâncias dos componentes da velocidade do vento em tempo real.

Os parâmetros d e $z_{0}$ são influenciados também pela estrutura física da vegetação (altura, forma e flexibilidade das plantas; tamanho e arranjo dos ramos e folhas) e por sua distribuição espacial pela área (SHAW \& PEREIRA, 1982). Algumas equações analíticas, baseadas nas interpretações físicas dos parâmetros de rugosidade, e outras empíricas, relacionam essas características e a transferência de momentum com a rugosidade da superfície (LETTAU, 1969; RAUPACH, 1992, 1994; MacDONALD et al., 1998), sendo alternativas aos métodos baseados na eq. (1). Entretanto, a mudança no arrasto efetivo de cada elemento rugoso, com incremento relativo da proteção aerodinâmica entre os elementos, torna esses modelos aplicáveis somente em situações de moderada separação dos elementos, como no caso de vegetação esparsa (LLOYD et al., 1992; SHAW \& PEREIRA, 1982).

Para diversas superfícies vegetadas tem-se observado que $\mathrm{z}_{0} / \mathrm{h}$ varia com o índice de área foliar (SHAW \& PEREIRA, 1982; THOM, 1971). A taxa de variação pode ser representada pela relação $\mathrm{z}_{0} / \mathrm{h}=\lambda(1-\mathrm{d} / \mathrm{h})$, sugerida por Thom (1971), em que, $\mathrm{h}$ é altura do dossel e $\lambda$ é um coeficiente de proporcionalidade. Relação linear entre $\mathrm{z}_{0} \mathrm{e}(\mathrm{h}-\mathrm{d})$ e proporcional a $\lambda=0,29$ foi observado por Pereira \& Shaw (1982), para extensa faixa de densidade do dossel. Porém, para dosséis esparsos a relação não foi constante, sendo dependente da distribuição vertical e da densidade do dossel. Para essas condições, $\lambda$ pode ser determinado pela estrutura física da vegetação (RAUPACH, 1992, 1994; MacDONALD et al., 1998).

A relação de Thom (1971) pode ser combinada com a lei logarítmica do vento (eq. 1) para estimar os parâmetros de rugosidade. Dessa forma, têm-se duas equações para estimar três parâmetros, diferente do método convencional, em que, apenas uma equação é utilizada. Esta abordagem mostra-se também alternativa às que aplicam $u_{*}$ na solução da lei logarítmica, visto que a determinação de $\lambda$ é mais simples do que $u_{*}$, além de serem apresentados na literatura valores para diversas culturas agrícolas e vegetação natural (WIERINGA, 1993). A combinação da relação de Thom (1971) e a lei logarítmica 
aplica-se à vegetação homogênea, o que não acontece com os métodos baseados apenas na relação entre transferência de momentum e características físicas da vegetação.

Considerando as dificuldades de estimativa de $\mathrm{d}$ e $\mathrm{z}_{0}$ para superfícies com vegetação esparsa e de porte alto, os objetivos do presente trabalho são: propor um método para determinar $\mathrm{z}_{0}$ e d, que combina a eq. (1) e a relação sugerida por Thom (1971) e avaliar as estimativas de $\mathrm{d}$ e $\mathrm{z}_{0}$ pelos métodos: i) convencional; ii) combinação do convencional com $\mathrm{u}_{*}$ determinada por correlações de vórtices turbulentos e iii) baseados na relação entre estrutura física (dimensões) da vegetação e a transferência de momentum.

\section{MATERIAL E MÉTODOS}

\subsection{Aspectos físicos da área experimental}

Os experimentos micrometeorológicos e da vegetação foram conduzidos no subsítio Fallow Bush do super-sítio Sul (SS) do HAPEX-Sahel (Hydrological and Atmospheric Pilot Experiment in the Sahel). O HAPEX-Sahel foi conduzido numa região semi-árida de savana, no Niger, oeste da África. A área experimental $(0,8 \times 1,0 \mathrm{~km})$ era limitada ao norte, nordeste e noroeste por áreas de Tiger Bush com altura da vegetação entre 4 e $6 \mathrm{~m}$ e, nas outras direções, por campos de milheto com altura média de 2,6 m (WALLACE et al., 1994). A área de Fallow Bush de SS apresentava vegetação escassa, dividida em duas camadas: uma, formada por vegetação rasteira de gramíneas e herbáceas e outra, composta por arbustos lenhosos distribuídos aleatoriamente e com predominância de Guiera senegalensis L., mas com algumas Combretum micranthum G. Don (LEVY \& JARVIS, 1999).

Análises da estrutura física de 220 arbustos de Guiera senegalensis L. foram feitas em fevereiro de 1992, em uma área de $6.750 \mathrm{~m}^{2}$. O ponto central da área estava a $120 \mathrm{~m}$ do mastro micrometeorológico. Determinou-se a máxima altura $(h$, em $\mathrm{m}$ ), a altura do raio máximo $\left(\mathrm{h}_{\text {máx }}, \mathrm{em} \mathrm{m}\right)$ e o raio máximo $\left(\mathrm{r}_{\text {máx }}\right.$, em $\left.\mathrm{m}\right)$ da copa. Avaliações mensais semidiretas do índice de área foliar $\left(I A F, \mathrm{em} \mathrm{m}^{2} \mathrm{~m}^{-2}\right)$ e do índice de área superficial dos galhos ( $I A G, \mathrm{em} \mathrm{m}^{2} \mathrm{~m}^{-2}$ ) foram feitas entre junho e outubro de 1992 (LEVY \& JARVIS, 1999). Havia cerca de 327 arbustos/ha de Guiera senegalensis L., com h $=2,06 \pm 0,47 \mathrm{~m}$ e $\mathrm{r}_{\text {máx }}=1,57 \pm 0,56 \mathrm{~m}$. Aárea projetada ocupada pelos arbustos representou cerca de $29 \%$ da área total de Fallow Bush.

\subsection{Medidas micrometeorológicas}

Medidas micrometeorológicas foram obtidas entre 20/08 e 30/09/92, no período de transição da estação chuvosa para a seca (WALLACE et al., 1994). O perfil de velocidade horizontal do vento foi medido por quatro anemômetros de canecas (A100H, VECTOR INSTRUMENTS, North Wales, UK) dispostos a 3,0; 4,1; 5,3 e 8,5 $\mathrm{m}$ acima do solo. O saldo de radiação $\left(\mathrm{R}_{\mathrm{n}}\right)$ foi medido por dois saldos radiômetros (CN1R, MIDDLETON SOLAR, Victoria, AU), sendo um sobre os arbustos e outro acima das gramíneas/herbáceas, ambos a $3 \mathrm{~m}$ acima do solo. O fluxo de calor no solo $(G)$ foi medido por oito placas de fluxo de calor em pares (CN3, MIDDLETON SOLAR, Victoria, AU) a 0,05 m de profundidade abaixo de cada tipo de vegetação. Considerou-se a média de todas as medidas como fluxo de calor no solo. As medidas foram feitas em intervalos de $30 \mathrm{~s}$ e suas médias armazenadas a cada 10 min por um datalogger (CR21X, CAMPBELL SCIENTIFIC Inc., Logan, UT).

Os componentes vertical (w), longitudinal (u) e latitudinal (v) da velocidade do vento foram medidas por anemômetro sônico tridimensional (GILL R3, SOLENT, Southampton, UK). A temperatura do $\operatorname{ar}(\mathrm{T})$ foi medida por um termômetro resistivo de platina (Department of Meteorology, UNIVERSITY OF READING, Reading, UK), e um higrômetro a infravermelho (IR-2000, OPHIR CORPORATION, Lakewood, CO) media a umidade do ar (q). Estes instrumentos estavam no topo de um mastro e a $9 \mathrm{~m}$ acima do solo. As variâncias e covariâncias médias entre as flutuações das variáveis supracitadas foram calculadas a cada 10 min de medidas em freqüência de $20 \mathrm{~Hz}$ e armazenadas em datalogger (WALLACE et al., 1994). Correções para a rotação das coordenadas, freqüência de resposta limitada dos sensores, média do comprimento do trajeto, separação lateral e longitudinal do sensor e freqüência de resposta do sistema de aquisição de dados foram feitas conforme apresentado por Lloyd et al. (1997).

\subsection{Estimativas dos parâmetros de rugosidade aerodinâmica da superfície}

Nas determinações dos parâmetros de rugosidade da superfície utilizaram-se médias de $30 \mathrm{~min}$ de todos os elementos micrometeorológicos, como recomendado por Monteith \& Unsworth (1990), e as médias das características físicas da vegetação.

\subsubsection{Método convencional}

$\mathrm{Na}$ abordagem convencional, $\mathrm{d}$ e $\mathrm{z}_{0}$ foram estimados por regressão linear simples de $U_{z}$ em função de $\ln (z-d)$, variandose $d$ até que o coeficiente de determinação $\left(r^{2}\right)$ fosse máximo (PEREIRA, 2002). Posteriormente, $\mathrm{u}_{*} \mathrm{e} \mathrm{z}_{0}$ foram determinados pelos valores do intercepto (a) e da inclinação (b) da regressão linear com maior $\mathrm{r}^{2}$, pelas seguintes relações analíticas:

$$
\begin{aligned}
& \mathrm{u}_{*}=\mathrm{kb} \\
& \mathrm{z}_{0}=\exp \left(-\frac{\mathrm{a}}{\mathrm{b}}\right)
\end{aligned}
$$

em que, $\mathrm{k}(=0,40)$ é a constante de von Kármán. 
2.3.2. Métodos baseados na combinação do convencional com a velocidade de friç̧ão medida por correlação de vórtices turbulentos

\subsubsection{Método de Lloyd}

Pelo método de Lloyd (LLOYD et al., 1992), os valores de $\mathrm{d}$ e $\mathrm{z}_{0}$ foram determinados pela regressão linear entre $(\mathrm{z}-\mathrm{d}) /$ $z_{0}$ e as respectivas alturas de medida $z$, em que o intercepto da regressão forneceu o valor de $\mathrm{d}$ e a inclinação o de $\mathrm{z}_{0}$. A relação $(z-d) / z_{0}$ foi obtida diferenciando a eq. (1) em função de $u_{*} e$ aplicando-se a exponencial em ambos os lados da equação, o que resultou na seguinte expressão:

$$
\frac{\mathrm{z}-\mathrm{d}}{\mathrm{z}_{0}}=\exp \left[\mathrm{k}\left(\frac{\mathrm{dU_{ \textrm {z } }}}{\mathrm{du}_{*}}\right)\right]
$$

A taxa de variação dUz/du* foi estimada para cada altura de medida da velocidade do vento como sendo a inclinação da regressão linear de $\mathrm{U}_{\mathrm{z}}$ em função de $\mathrm{u}_{*}$ forçada a passar pela origem, como sugerido por Lloyd et al. (1992). Utilizou-se na determinação da inclinação da regressão $u_{*}$ determinado por correlação de vórtices turbulentos $\left(\mathrm{u}_{*_{\mathrm{e}}}\right) . \mathrm{u}_{*_{\mathrm{e}}}$ foi estimada pelas flutuações dos componentes longitudinal ( $\left.u^{\prime}\right)$ e vertical $\left(w^{\prime}\right)$ da velocidade do vento, i.e.:

$$
\mathrm{u}_{*_{\mathrm{e}}}={\overline{\left(-\mathrm{u}^{\prime} \mathrm{w}^{\prime}\right)^{0,5}}}^{0,5}
$$

\subsubsection{Método de Takagi}

O método de Takagi (TAKAGI et al., 2003) é similar ao convencional, porém utiliza $u_{*_{e}}$ na solução da lei logarítmica. Por essa abordagem, a e b foram determinados para cada valor de $d$, gerando um valor para a velocidade de fricção pelo perfil $\left(\mathrm{u}_{* \mathrm{p}}\right)$. O processo foi interrompido quando $\mathrm{u}_{*_{\mathrm{p}}} \approx \mathrm{u}_{*_{\mathrm{e}}}$, determinando o valor de $\mathrm{d}$, enquanto $\mathrm{z}_{0}$ foi fornecido pela eq. 3

\subsubsection{Métodos derivados da relação entre estrutura física da vegetação e a transferência de momentum}

\subsubsection{Método de Raupach}

Neste método, d foi calculado pela equação empírica ajustada a medidas em túnel de vento em função do índice de área do dossel ( $\Lambda$ ), na forma (RAUPACH, 1994):

$$
1-\frac{\mathrm{d}}{\mathrm{h}}=\frac{1-\exp (-\sqrt{7,5 \Lambda})}{\sqrt{7,5 \Lambda}}
$$

O índice de área do dossel foi calculado como a área total de um único lado de todos os elementos do dossel (folhas e galhos) pela área unitária de solo, ou seja, $\Lambda=\mathrm{IAF}+(\mathrm{IAG} / 2)$. Utilizou-se a média dos valores de IAF e IAG avaliados de setembro a outubro de 1992.
$\mathrm{Na}$ estimativa de $\mathrm{z}_{0}$ foi utilizado o perfil logaritmo do vento (eq. 1) com correções para a subcamada rugosa. Esse perfil quando aplicado $\mathrm{a} \mathrm{z}=\mathrm{h}$ apresenta a seguinte forma (RAUPACH, 1992, 1995):

$$
\frac{\mathrm{z}_{0}}{\mathrm{~h}}=\left(1-\frac{\mathrm{d}}{\mathrm{h}}\right) \exp \left(\Psi_{\mathrm{h}}-\mathrm{k} \gamma\right)
$$

em que, $\Psi_{\mathrm{h}}$ é uma função de influência na forma do perfil, que quantifica o desvio do coeficiente atual de difusão turbulenta para momentum dentro da subcamada rugosa $\mathrm{z} z=\mathrm{h} ; \gamma=\mathrm{U}_{\mathrm{h}} / \mathrm{u}_{*}$ e $\mathrm{U}_{\mathrm{h}}$ é a velocidade do vento a $\mathrm{z}=\mathrm{h}$.

Na determinação de $\Psi_{\mathrm{h}}$ utilizou-se a aproximação de Raupach (1992) e Raupach (1995), ou seja:

$$
\Psi_{\mathrm{h}}=\ln \left(\mathrm{c}_{\mathrm{w}}\right)-1+\mathrm{c}_{\mathrm{w}}^{-1}
$$

em que, $c_{w}=2$, como sugerido por Raupach (1995), resultando em $\Psi_{\mathrm{h}}=0,1932$.

A relação $\mathrm{U}_{\mathrm{h}} / \mathrm{u}_{*}=\gamma$ foi calculada como função implícita do índice de área frontal ou densidade de rugosidade $\left(\lambda_{\mathrm{f}}\right)$, expressa na forma (RAUPACH, 1995):

$$
\frac{\mathrm{U}_{\mathrm{h}}}{\mathrm{u}_{*}}=\gamma=\frac{\exp \left(\mathrm{c} \lambda_{\mathrm{f}} \gamma / 2\right)}{\sqrt{\mathrm{C}_{\mathrm{s}}+\mathrm{C}_{\mathrm{R}} \lambda_{\mathrm{f}}}}
$$

em que, $\lambda_{\mathrm{f}}\left(=\mathrm{n} \mathrm{D} \mathrm{h} / \mathrm{A}_{\mathrm{T}}\right)$ é o índice de área frontal, definido como a área da silhueta dos obstáculos, dividida pela área de solo ocupada pelos mesmos (área específica); $\mathrm{C}_{\mathrm{S}}(=0,003)$ é o coeficiente de arrasto do substrato; $C_{R}(=0,3)$ é o coeficiente de arrasto de um elemento rugoso isolado; c $(=0,37)$ é o coeficiente de proteção aerodinâmica; n $(=220)$ é o número de elementos rugosos ocupando a área total de solo $\mathrm{A}_{\mathrm{T}}\left(=6.750 \mathrm{~m}^{2}\right)$ e D (=2 $\mathrm{r}_{\text {máx }}$, em m) é o máximo diâmetro dos arbustos. A eq. (9) foi resolvida por método iterativo (RAUPACH, 1992).

\subsubsection{Método de MacDonald}

$\mathrm{Na}$ estimativa de $\mathrm{d}$ por esse método foi utilizada a equação empírica ajustada a medidas dos parâmetros de rugosidade da superfície em função do índice de área plana (MacDONALD et al., 1998), ou seja:

$$
\frac{\mathrm{d}}{\mathrm{h}}=1+\mathrm{A}^{-\lambda_{\mathrm{p}}}\left(\lambda_{\mathrm{p}}-1\right)
$$

em que, $\lambda_{\mathrm{P}}$ é o índice de área plana e $\mathrm{A}(=4,43$ para obstáculos dispostos aleatoriamente) é uma constante que controla a curvatura da função. $\lambda_{P}$ foi estimado como a razão da área projetada dos arbustos por $A_{T}$ (MacDONALD et al., 1998). Considerouse a área projetada como a área da circunferência dos arbustos calculada em função da média do seu raio máximo.

Nesse modelo a estimativa de $\mathrm{z}_{0}$ foi derivada do perfil logarítmico do vento, porém descartando a interferência das onduletas entre os elementos rugosos, ou seja, sem correções para a subcamada rugosa, isto é: 


$$
\frac{\mathrm{z}_{0}}{\mathrm{~h}}=\left(1-\frac{\mathrm{d}}{\mathrm{h}}\right) \exp \left[-\left(\lambda_{\mathrm{f}} \frac{\mathrm{C}_{\mathrm{R}}}{\mathrm{k}^{2}}\left(1-\frac{\mathrm{d}}{\mathrm{h}}\right)\right)^{-0,5}\right]
$$

com $C_{R}=0,6$, o dobro do valor utilizado por Raupach (1992).

\subsubsection{Método baseado na combinação do convencional com as características físicas da vegetação}

O método proposto no presente trabalho utilizou a lei logarítmica do perfil de vento e a relação sugerida por Thom (1971), sendo aqui denominado de método convencional-Thom. A relação de Thom (1971) pode ser escrita da seguinte forma:

$$
\mathrm{z}_{0}=\lambda(\mathrm{h}-\mathrm{d})
$$

Variando-se d, a cada passo da iteração, $\mathrm{z}_{0}$ foi estimado pelo intercepto da regressão linear entre $\ln (z-d)$ e $U_{z}$, sendo simultaneamente calculado pela eq. (12). O valor de $\mathrm{d}$ foi determinado quando convergiram os valores de $z_{0}$ estimados pelo perfil e pela eq. (12). Utilizaram-se três valores de $\lambda$ na estimativa dos parâmetros de rugosidade, sendo um valor médio $(\lambda=0,166 \pm 0,060)$ obtido de alguns trabalhos sobre vegetação esparsa de arbustos em região semi-árida (DOLMAN et al., 1992; MOLION \& MOORE, 1983; LLOYD et al., 1992), e os outros dois determinados em função da estrutura física da vegetação na área em estudo.

As equações baseadas na estrutura física foram obtidas dos modelos de MacDonald e Raupach. Igualando as eq. (7) e (12), obteve-se a relação para estimativa de $\lambda$ pelo modelo de Raupach:

$$
\lambda=\exp \left(\Psi_{\mathrm{h}}-\mathrm{k} \lambda\right)
$$

e igualando as eq. (11) e (12), determinou-se a equação derivada do modelo de MacDonald:

$$
\lambda=\exp \left[-\left(\lambda_{\mathrm{f}} \frac{\mathrm{C}_{\mathrm{R}}}{\mathrm{k}^{2}}\left(1-\frac{\mathrm{d}}{\mathrm{h}}\right)\right)^{-0,5}\right]
$$

\subsection{Condições de neutralidade atmosférica}

Os dados utilizados nas análises foram restritos a condições próximas da neutralidade atmosférica para satisfazer a lei do perfil logarítmico do vento. Foram selecionadas observações que apresentavam módulo da energia disponível $\left|\mathrm{R}_{\mathrm{n}}-\mathrm{G}\right|<20 \mathrm{~W} \mathrm{~m}^{-2}$ e do fluxo de calor sensível $|\mathrm{H}|<15 \mathrm{~W} \mathrm{~m}^{-2}$, similar a de Bruin \& Moore (1985). Devido aos erros proporcionados pela inércia dos anemômetros de canecas, perfis com velocidade do vento no anemômetro inferior menor que $1,0 \mathrm{~ms}^{-1} \mathrm{e} \mathrm{u}_{*}<0,1 \mathrm{~ms}^{-1}$ também foram excluídos, seguindo os procedimentos de Shuttleworth et al. (1988). Foram consideradas, apenas, observações com direção do vento entre $S E$ e $S S E$, devido à bordadura inadequada para o nível superior $(8,5 \mathrm{~m})$ de medida nas outras direções (LYRA, 2005).
Sendo as condições acima satisfeitas, avaliou-se a estabilidade atmosférica no nível de medida do anemômetro sônico $(9 \mathrm{~m})$ pelo parâmetro $\zeta$ de estabilidade (MONIN \& OBUKHOV, 1954), em que só foram utilizadas observações que apresentaram módulo de $\zeta<0,0325$. O parâmetro $\zeta$ foi estimado pela seguinte relação:

$$
\zeta=\frac{\mathrm{z}}{\mathrm{L}}=\frac{\mathrm{zkg} \overline{\mathrm{w} \cdot \theta}}{\mathrm{u}_{*}^{3} \theta}
$$

em que, L (em m) é a escala de comprimento (altura) de MoninObukhov; $\mathrm{g}\left(=9,81 \mathrm{~m} \mathrm{~s}^{-2}\right)$ é a aceleração devido a gravidade; $\theta(\mathrm{em} \mathrm{K})$ é a temperatura potencial; e $\overline{\mathrm{w}^{\prime} \theta}{ }^{\prime}\left(\mathrm{em} \mathrm{m} \mathrm{s}^{-1} \mathrm{~K}\right)$ é a covariância média entre as flutuações do componente vertical do vento e da temperatura potencial do ar.

\subsection{Análise estatística}

Na avaliação da influência de d e $\mathrm{z}_{0}$ na estimativa da velocidade horizontal do vento pela eq. (1), os seguintes coeficientes estatísticos foram utilizados: média aritmética, desvio padrão e coeficiente de determinação $\left(r^{2}\right)$, intercepto (a) e coeficiente angular (b) da regressão linear entre $\mathrm{U}_{\mathrm{z}}$, medido (X) e estimado pela lei logarítmica do vento $(\mathrm{Y})$. A velocidade horizontal do vento foi estimada para cada nível de medida $(3,0 ; 4,1 ; 5,3 \mathrm{e}$ $8,5 \mathrm{~m}$ ), utilizando $\mathrm{u}_{*}$ determinado por correlação de vórtices turbulentos $(9 \mathrm{~m})$, totalizando quatro amostras por horário para cada método.

Utilizou-se também a raiz do quadrado médio do erro (RQME, em m s${ }^{-1}$ ), como descrito por Willmott (1981). Avaliou-se a concordância entre os valores observados e estimados pelo perfil logarítmico para os valores de $\mathrm{de} \mathrm{z}_{0}$ determinados pelos diversos métodos, utilizando o índice de concordância de Willmott (I) (WILLMOTT, 1981). Efetuaram-se os testes t e F para as médias e variâncias de $\mathrm{U}_{\mathrm{z}}$, respectivamente, estimados em função de $\mathrm{d}$ e $\mathrm{z}_{0}$ determinados pelos métodos analisados e para os valores medidos.

\section{RESULTADOS E DISCUSSÃO}

\subsection{Parâmetros de rugosidade da superfície}

Na região do Sahel são difíceis de serem observadas condições de neutralidade atmosférica, em função do elevado fluxo de calor sensível, com forte turbulência originada por flutuação térmica e a baixas velocidades do vento, inibindo a geração da energia mecânica turbulenta (DE BRUIN \& VERHOEF, 1997). Foram obtidas 600 observações médias de 30 min com medidas simultâneas do perfil da velocidade horizontal do vento e correlação de vórtices turbulentos, mas apenas seis delas $(1,0 \%)$ tinham condições próximas à neutralidade $\mathrm{e}$ bordadura adequada. 
A relação entre $U_{z}$ e $\ln (z-d)$ apresentou ajuste estatístico satisfatório do perfil para d calculado a cada observação pelo método convencional ( $\mathrm{CO})$, com coeficiente de determinação superior a 0,99, indicado por Takagi et al. (2003) como limite mínimo aceitável. Com esse método obteve-se $\mathrm{d}=1,09 \pm 0,14 \mathrm{~m}$ e $\mathrm{z}_{0}=0,184 \pm 0,017 \mathrm{~m}$ (Tabela 1$)$. O coeficiente de variação (cv) foi de $13 \%$ para $\mathrm{d}$, e $9,5 \%$ para $\mathrm{z}_{0}$, sendo similares aos valores observados por Munro \& Oke (1973) para a cultura do trigo.

Para o método de Takagi (TA), a regressão linear de $\mathrm{U}_{\mathrm{z}}$ em função de $\ln (\mathrm{z}-\mathrm{d})$ apresentou $\mathrm{r}^{2}>0,99$ para todas as observações, enquanto para o convencional-Thom (CT), independente do valor de $\lambda$, um caso mostrou $r^{2}<0,99$, sendo desconsiderado das análises para este método. Pelo TA obtevese $d=1,07 \pm 0,35 \mathrm{~m} \mathrm{e}_{0}=0,190 \pm 0,065 \mathrm{~m}$. Pelo CT, $d$ variou entre $1,12 \pm 0,40 \mathrm{~m}, \operatorname{com} \lambda=0,190$, estimado pela relação de MacDonald $\left(\mathrm{CT}_{\mathrm{MD}}\right)$, e $1,40 \pm 0,05 \mathrm{~m}$ em função de $\lambda=0,166$ médio da literatura $\left(\mathrm{CT}_{\mathrm{LT}}\right)$, enquanto $\mathrm{z}_{0}$ variou de $0,179 \pm 0,075$ $\mathrm{m}\left(\mathrm{CT}_{\mathrm{MD}}\right)$ a $0,109 \pm 0,009 \mathrm{~m}\left(\mathrm{CT}_{\mathrm{LT}}\right)$.

$\mathrm{O} \mathrm{CT}_{\mathrm{LT}}$ apresentou cv para os parâmetros de rugosidade inferiores aos determinados pelo $\mathrm{CO}$ e de $3,7 \%$ para de de $7,9 \%$ para $\mathrm{z}_{0}$. Nos demais métodos, baseados no convencional, e que utilizaram $\mathrm{u}_{*}$ determinado por correlação de vórtices ou $\lambda$, os valores de cv foram superiores, e entre $32,6 \%$ (TA) e $35,4 \%$ $\left(\mathrm{CT}_{\mathrm{MD}}\right)$ para d e no intervalo de $34,0 \%$ (TA) a $42,2 \%\left(\mathrm{CT}_{\mathrm{MD}}\right)$ para $\mathrm{z}_{0}$. Contrário ao $\mathrm{CO}$, nesses métodos os $\mathrm{cv}$ para $\mathrm{z}_{0}$ foram superiores aos de d. Takagi et al. (2003) determinaram valor de cv de até $85,7 \%$ para $\mathrm{z}_{0}$, não sendo apresentado os resultados para d.

Apesar de empregar duas equações na iteração (CT), ou soluções simples do perfil em função de $\mathrm{u}_{*}$ (TA) na estima- tiva de $\mathrm{d}$ e $\mathrm{z}_{0}$, os métodos CT e TA mostraram-se sensíveis a variações em $\lambda$ ou $u_{*}$, sendo esses fontes potenciais de erro na determinação dos parâmetros de rugosidade. d estimado pelo TA foi menos sensível a variações em $u_{*}$ do que $z_{0}$. Padrão análogo foi mostrado para $\lambda$ no método convencional-Thom. Porém, os parâmetros de rugosidade tiveram tendência contrária, em que $\mathrm{d}$ variou inversamente proporcional a $\mathrm{u}_{*}$ ou $\lambda$ e $\mathrm{z}_{0}$ diretamente. Variações de $10 \%$ em $u_{*}$ resultaram em diferenças de $-21,8 \%$ em d e de $40,1 \%$ em $z_{0}$ pelo TA, enquanto a mesma variação em $\lambda$ para CT , proporcionou variações similares e de $-22,1 \%$ para d e de $43,8 \%$ para $z_{0}$.

No método de Lloyd (LO) utilizaram-se as seis observações consideradas em $\mathrm{CO}$ e no TA, pela impossibilidade de avaliação do ajuste do perfil ao valor de d a cada horário. Pelo LO, d é calculado como o intercepto da regressão entre $\mathrm{z}$ e $(z-d) / z_{0}$, sendo igual a $0,95 \pm 0,27 \mathrm{~m}$, e $z_{0}=0,204 \pm 0,012$ $m$ determinado pela inclinação da regressão (Figura 1). O erro padrão de estimativa de $\mathrm{d}$ e $\mathrm{z}_{0}$ padronizados pelos seus valores foram de 0,28 e 0,059 , respectivamente, sendo inferior ao determinado por Lloyd et al. (1992) para d $(0,38)$ e o mesmo para $z_{0}$ $(0,059)$. As diferenças no erro padrão padronizado para $\mathrm{d}$ podem estar associadas à extensa faixa de estabilidade atmosférica considerada por esses autores como sendo próxima à neutra $(|\zeta|<0,256)$, ou a medidas realizadas no interior da subcamada rugosa (DOLMAN et al. 1992).

Pelo $\mathrm{CT}_{\mathrm{LT}} \mathrm{d}$ foi em média 22,7\% superior aos determinados pelos demais métodos que utilizaram medidas do perfil de vento, enquanto $z_{0}$ foi 69,9\% inferior. Quando desconsiderado $\mathrm{CT}_{\mathrm{LT}}$ das avaliações, os métodos baseados no perfil tiveram extremos para d com diferença de $16,6 \%$ e de $15,5 \%$ para $z_{0}$.

Tabela 1 - Deslocamento do plano zero (d) e comprimento de rugosidade $\left(\mathrm{z}_{0}\right)$ estimados pelos métodos baseados na lei logarítmica do vento e pelos métodos em função da estrutura física da vegetação. Deslocamento do plano zero normalizado pela altura do dossel $(\mathrm{d} / \mathrm{h})$ e relação entre comprimento de rugosidade $\left(\mathrm{z}_{0}\right)$ e a diferença $(\mathrm{h}-\mathrm{d})$. Valor entre parênteses representa o erro padrão de estimativa para o método de Lloyd e o desvio padrão para os demais métodos

\begin{tabular}{ccccc}
\hline Método $^{\mathbf{1}}$ & $\mathbf{d}(\mathbf{m})$ & $\mathbf{z}_{\mathbf{0}}(\mathbf{m})$ & $\mathbf{d} / \mathbf{h}$ & $\lambda=\mathbf{z}_{\mathbf{0}} /(\mathbf{h}-\mathbf{d})$ \\
\hline $\mathrm{CO}$ & $1,09( \pm 0,14)$ & $0,184( \pm 0,017)$ & 0,53 & 0,189 \\
$\mathrm{CT}_{\mathrm{RP}}$ & $1,14( \pm 0,38)$ & $0,173( \pm 0,072)$ & 0,56 & 0,188 \\
$\mathrm{CT}_{\mathrm{MD}}$ & $1,12( \pm 0,40)$ & $0,179( \pm 0,075)$ & 0,54 & 0,190 \\
$\mathrm{CT}_{\mathrm{LT}}$ & $1,40( \pm 0,00)$ & $0,109( \pm 0,001)$ & 0,68 & 0,166 \\
$\mathrm{TA}$ & $1,07( \pm 0,35)$ & $0,190( \pm 0,065)$ & 0,52 & 0,193 \\
$\mathrm{LO}$ & $0,95( \pm 0,27)$ & $0,204( \pm 0,012)$ & 0,46 & 0,185 \\
$\mathrm{RP}$ & 1,10 & 0,181 & 0,53 & 0,189 \\
$\mathrm{MD}$ & 1,12 & 0,180 & 0,54 & 0,191 \\
\hline
\end{tabular}

${ }^{(1)}$ Método convencional (CO); convencional-Thom utilizando $\lambda$ estimado pela relação de Raupach (1992) $\left(\mathrm{CT}_{\mathrm{RP}}\right)$; com $\lambda$ determinado pela relação de MacDonald et al. (1998) $\left(\mathrm{CT}_{\mathrm{MD}}\right)$ e com $\lambda$ médio da literatura $\left(\mathrm{CT}_{\mathrm{LT}}\right.$ ); método de Takagi (TA); Lloyd (LO); Raupach (RP) e MacDonald (MD). 
A vegetação mostrou baixa densidade de rugosidade ou índice de área frontal $\left(\lambda_{\mathrm{f}}=0,21\right)$, sendo desprezível a influência da proteção aerodinâmica entre os elementos rugosos no transporte de momentum. Para este valor de $\lambda_{\mathrm{f}}$, o modelo de Raupach (RP) pode ser aplicado (RAUPACH, 1994). Os métodos que consideram a estrutura física da vegetação apresentaram d de 1,10 e 1,12 m para os métodos de RP e MacDonald (MD), respectivamente e $\mathrm{z}_{0}$ de $0,181(\mathrm{RP})$ e $0,180 \mathrm{~m}(\mathrm{MD})$. Estes métodos tiveram diferença inferior a 2,0\% para d e a $1,0 \%$ para $\mathrm{z}_{0}$. Com exceção de $\mathrm{CT}_{\mathrm{LT}}$, os parâmetros de rugosidade determinados pelas medidas do perfil foram no mesmo intervalo dos calculados em função da estrutura física da vegetação.

$\mathrm{O}$ deslocamento do plano zero normalizado pela altura dos arbustos $(\mathrm{d} / \mathrm{h})$ variou entre $0,46(\mathrm{LO})$ e $0,68\left(\mathrm{CT}_{\mathrm{LT}}\right)$ (Tabela 1). Estes valores foram inferiores ao determinado sobre superfície esparsa, composta por arbustos, eucaliptos e uma camada de grama seca $(0,75)$, na Austrália (GARRATT, 1980). Molion \& Moore (1983), utilizando um procedimento analítico baseado na conservação de massa do perfil, determinaram $\mathrm{d} / \mathrm{h}=0,65$ para as mesmas medidas de Garratt (1980), sendo próximo ao estimado pelo $\mathrm{CT}_{\mathrm{LT}}$. Lloyd et al. (1992), na região do Sahel, sobre cobertura vegetal similar à do presente trabalho (predominância de Guiera senegalensis L.), porém no período seco, apresentaram valor inferior e de $0,40 \mathrm{~h}$. Este valor mostrou concordância com o observado pelo LO.

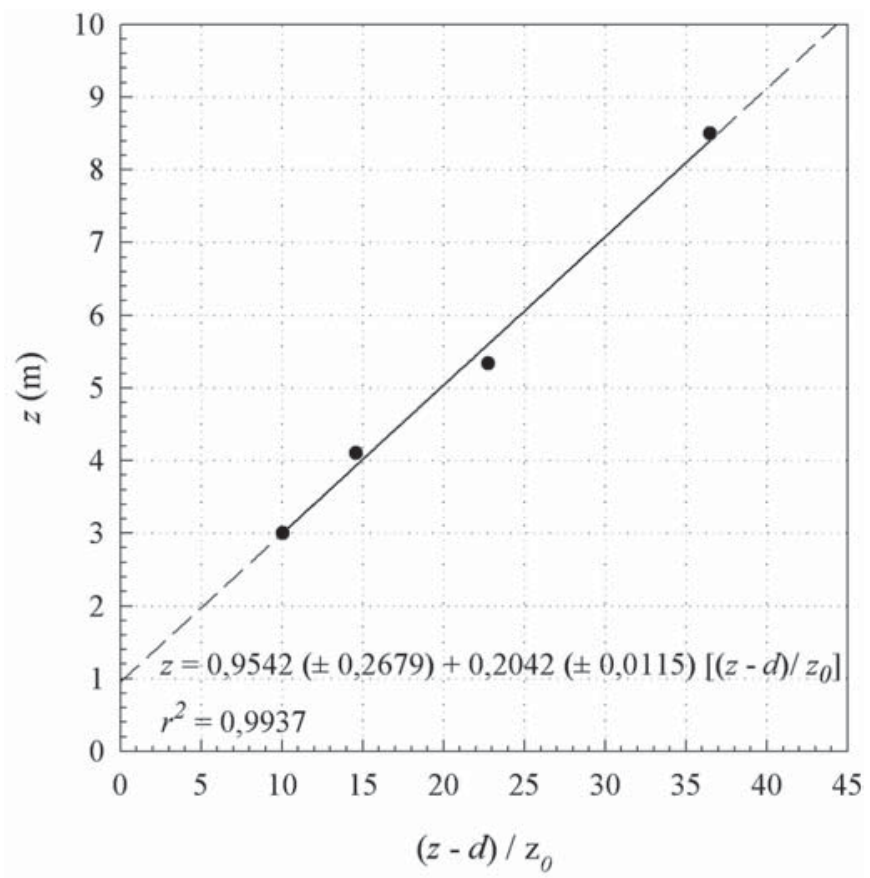

Figura 1 - Relação entre altura de medida $(z)$ da velocidade horizontal do vento $\left(\mathrm{U}_{\mathrm{z}}\right)$ em função de $(\mathrm{z}-\mathrm{d}) / \mathrm{z}_{\mathrm{o}}$ derivado da inclinação da regressão entre a velocidade de fricção determinada por correlação de vórtices e $\mathrm{U}_{\mathrm{z}}$ forçada pela origem para cada altura de medida. Valores entre parênteses representam o erro padrão de estimativa do intercepto (d) e da inclinação $\left(z_{o}\right)$ da regressão.
Dolman et al. (1992) comentam que para superfície esparsa, formada por arbustos e árvores (Tiger Bush) no Sahel, o deslocamento do plano zero de $0,57 \mathrm{~h}$ mostra-se representativo da superfície subjacente. Para vegetação similar no Sahel, De Bruin \& Verhoef (1997) utilizando as relações de similaridade para convecção livre, estimaram deslocamento do plano zero normalizado de 0,53 . Baseado nas análises de Shaw \& Pereira (1982), e considerando a altura do máximo diâmetro do dossel $\left(\mathrm{h}_{\text {máx }}=1,49 \pm 0,47 \mathrm{~m}\right)$ como a de sua máxima densidade, para um índice de área do dossel $(\Lambda)$ medido de 0,428 , obteve-se $\mathrm{d}=0,55 \mathrm{~h}$. Esses valores apresentam concordância com os estimados no presente trabalho, com exceção de $\mathrm{CT}_{\mathrm{LT}}$ e de $\mathrm{LO}$ que discordaram do valor obtido por Dolman et al.(1992).

$\mathrm{O}$ índice de área frontal das árvores $\left(\lambda_{\mathrm{f}}=0,20\right)$ para as condições de Garratt (1980) foi similar ao observado no presente trabalho, ou seja, densidade da vegetação análoga. O valor superior de d/h apresentado por Garratt (1980), e em menor proporção pelo recalculado por Molion \& Moore (1983), podem ter sido influenciados pela distribuição vertical da densidade foliar das árvores de eucaliptos, as quais mostram maior densidade próxima ao topo do dossel, o que contribui para o deslocamento de $\mathrm{z}_{0}$ superior (SHAW \& PEREIRA, 1982). De qualquer forma, o valor de Garratt (1980) foi considerado elevado por Lloyd et al. (1992) para $\lambda_{\mathrm{f}}$ observado.

O inferior determinado por Lloyd et al. (1992) justificase, em parte, pelas medidas terem sido realizadas no período seco na região do Sahel, em que o índice de área foliar dos arbustos mostra-se inferior ao observado na estação chuvosa (LEVY \& JARVIS, 1999). Considerando-se que d é o nível médio de absorção de momentum (THOM, 1971), e que a diminuição da densidade de folhagem resulta em menor área de sumidouro de momentum, espera-se diminuição de d.

$O$ coeficiente de proporcionalidade $\lambda\left[=\mathrm{z}_{0} /(\mathrm{h}-\mathrm{d})\right]$ foi no intervalo de 0,166 ( $\left.\mathrm{CT}_{\mathrm{LT}}\right)$ a 0,193 (TA). No CT, as diferenças entre $\lambda$ médio da literatura e os observados para os demais métodos foi em média $14,4 \%$, enquanto que para os valores estimados pelas relações analíticas de MacDonald $(0,188)$ e Raupach $(0,190)$ essas diferenças foram inferiores a $0,4 \%$, ou seja, a determinação de $\lambda$ em função das características físicas da vegetação mostrou-se satisfatória. Para dosséis esparsos $(\Lambda<1,0), \lambda$ apresenta alta variação em função da densidade e distribuição vertical do dossel (SHAW \& PEREIRA, 1982). Dessa forma, as diferenças elevadas entre $\lambda$ da literatura e o dos métodos avaliados podem ter sido resultados da variação entre a densidade e a distribuição vertical da vegetação da área em estudo e as avaliadas na literatura, apesar de considerados apenas valores da literatura, determinados para vegetação esparsa de arbustos em região semi-árida (DOLMAN et al., 1992; MOLION \& MOORE, 1983; LLOYD et al., 1992). 
Para cultura artificial, $\lambda$ mostrou valor de 0,36 (THOM, 1971), sendo de 0,25 para floresta de pinus (MOLION \& MOORE, 1983). Para dosséis densos $(\Lambda>1)$, Shaw \& Pereira (1982) determinaram relação linear entre $\mathrm{z}_{0} \mathrm{e}(\mathrm{h}-\mathrm{d})$ e proporcional a 0,29 . Contudo, para dosséis esparsos $(\Lambda<1,0)$ essa proporção foi inferior e não constante.

Tendência análoga à observada por Garratt (1980) para $\mathrm{d} / \mathrm{h}$ foi mostrada por $\lambda$, com valor superior aos dos métodos analisados, e de 0,38 . O valor de $\lambda$ apresentado por Garratt (1980) foi elevado para a densidade de vegetação observada, resultado principalmente da influência das árvores de eucalipto no valor de $\mathrm{d}$.

Dolman et al. (1992) utilizaram o método de Lloyd para estimar o comprimento de rugosidade, obtendo $\mathrm{z}_{0}=0,21(\mathrm{~h}-\mathrm{d})$. O valor de $\lambda$ em função dos resultados de Shaw \& Pereira (1982) foi análogo ao de Dolman et al. (1992) e aproximadamente 0,21, enquanto o apresentado por Molion \& Moore (1983) foi de 0,169 . Esses valores foram próximos aos observados no presente trabalho, com exceção de $\mathrm{CT}_{\mathrm{LT}}$, que divergiu dos determinados por Shaw $\&$ Pereira (1982) e Dolman et al. (1992). Enquanto $\lambda$ determinado por Lloyd et al. (1992) foi inferior e de 0,124. O menor IAF para as condições de Lloyd et al. (1992) resultou em baixo valor de $\mathrm{d} / \mathrm{h}$, como comentado anteriormente, e de $\mathrm{z}_{0}$, que combinados, proporcionaram $\lambda$ inferior ao dos métodos analisados.

\subsection{Estimativas de $U_{z}$}

A média da velocidade do vento observada $\left(\overline{\mathrm{U}}_{\mathrm{z}_{-} \text {obs }}\right)$ nos quatro níveis de medida e nos seis horários considerados foi de 3,32 $\pm 1,06 \mathrm{~m} \mathrm{~s}^{-1}$, enquanto que a média da velocidade do vento estimada $\left(\overline{\mathrm{U}}_{\mathrm{z} \text { est }}\right)$ pela lei logarítmica do vento (eq. 1) em função de $\mathrm{d}$ e $\mathrm{z}_{0}$ determinados pelos métodos analisados e $\mathrm{u}_{*}$ dada por correlação de vórtices variou de $3,29 \pm 1,15 \mathrm{~m} \mathrm{~s}^{-1}$ (LO) a 3,83 $\pm 1,34 \mathrm{~m} \mathrm{~s}^{-1}\left(\mathrm{CT}_{\mathrm{LT}}\right)$ (Tabela 2). $\overline{\mathrm{U}}_{\mathrm{z} \text { est }}$ em função dos parâmetros de rugosidade de TA não apresentou diferença em relação ao valor médio observado. Para os outros métodos, a tendência geral foi de subestimativa de $\overline{\mathrm{U}}_{\mathrm{z} \_ \text {est }}(0,9 \%)$ com os parâmetros de rugosidade de $\mathrm{LO}$ e superestimativa entre 1,2\% (CO) e 15,4\% $\left(\mathrm{CT}_{\mathrm{LT}}\right)$. Apenas $\overline{\mathrm{U}}_{\mathrm{z} \text { est }}$ por $\mathrm{CT}_{\mathrm{LT}}$ mostrou diferença estatística significativa pelo teste-t $(\mathrm{p}<0,05)$ em relação a $\overline{\mathrm{U}}_{\mathrm{z} \_ \text {obs. }}$.

A raiz do quadrado médio do erro (RQME) da velocidade do vento estimada ( $U_{z}$ est $)$ em função de $d$ e $z_{0}$ dos métodos que utilizaram $u_{*}$ determinada por correlação foi inferior a observada para os demais métodos, sendo de $0,22 \mathrm{~m} \mathrm{~s}^{-1}$ para $\mathrm{LO}$ e de $0,23 \mathrm{~m} \mathrm{~s}^{-1}$ para TA. O maior RQME foi obtido quando utilizado $\mathrm{de} \mathrm{z}_{0}$ estimados pelo $\mathrm{CT}_{\mathrm{LT}}\left(0,62 \mathrm{~m} \mathrm{~s}^{-1}\right)$. RQME representou em torno de $6,7 \%$ de $\overline{\mathrm{U}}_{\mathrm{z} \_ \text {obs }}$ para $\mathrm{LO}$ e de $16,2 \%$ para $\mathrm{CT}_{\mathrm{LT}}$. Com exceção de $\mathrm{CT}_{\mathrm{LT}}$, RQME mostrou-se no mesmo intervalo (entre 0,22 e $0,26 \mathrm{~m} \mathrm{~s}^{-1}$ ) para $\mathrm{de} \mathrm{z}_{0}$ dos métodos baseados na estrutura física da vegetação e dos que utilizaram medidas do perfil de vento.
As regressões entre a velocidade do vento observada $\left(\mathrm{U}_{\mathrm{z}_{\mathrm{obs}}}\right)$ e $\mathrm{U}_{\mathrm{z}_{-} \text {sst }}$ mostraram interceptos não significativos estatisticamente $(p<0,05)$. Assim, utilizaram-se nas avaliações as regressões forçadas a passar pela origem (Figura 2). Os valores de $r^{2}$ acima de 0,9615 indicaram precisão satisfatória das estimativas. Este resultado diferiu do obtido por Righi (2004), em que $r^{2}<0,78$.

As estimativas de $\mathrm{U}_{\mathrm{z}}$ com base nos parâmetros de rugosidade de $\mathrm{CO}$ (Figura 2A), CT, independente de $\lambda$ (Figuras 2B, $2 \mathrm{C}$ e 2D), TA (Figura 2E) e os baseados na estrutura física da vegetação (Figuras $2 \mathrm{G} \mathrm{e} 2 \mathrm{H}$ ) mostraram tendência de superestimativa de $\mathrm{U}_{\mathrm{z}_{\text {_est }}}$ entre $0,9 \%$ (TA) e $16,2 \%\left(\mathrm{CT}_{\mathrm{LT}}\right)$. Padrão inverso foi apresentado por LO (Figura 2F), com subestimativa de $0,4 \%$ de $\mathrm{U}_{z_{-} \text {obs }}$. Esses valores encontram-se no intervalo de incerteza das medidas dos anemômetros de canecas ( 5\%) (WIERINGA, 1980), com exceção de $\mathrm{CT}_{\text {LT. }}$ Righi (2004) observou subestimativa entre 1 e $7 \%$ de $\mathrm{U}_{z_{-} \text {es }}$ em função de $\mathrm{d}$ e $\mathrm{z}_{0}$ determinados pelo método convencional.

O índice de concordância de Willmott (I) apresentou extremo inferior de 0,9334 para as estimativas em função dos parâmetros de $\mathrm{CT}_{\mathrm{LT}}$, sendo os superiores mostrados pelos métodos TA $(0,9894)$ e LO $(0,9896)$. Novamente, as estimativas utilizando os parâmetros de rugosidade da superfície pelo $\mathrm{CT}_{\mathrm{LT}}$ mostraram padrão divergente do apresentado pelos demais métodos e dos valores observados. Assim, $\mathrm{d} \mathrm{e}_{0}$ desse método não representaram

Tabela 2 - Média da velocidade horizontal do vento $\left(\bar{U}_{z}\right)$ e dos valores estimados pela lei logarítmica do perfil de vento em função dos parâmetros de rugosidade estimados pelos métodos baseados na lei logarítmica do vento e os em função da estrutura física da vegetação. Valores entre parênteses representam o desvio padrão da média. Raiz do quadrado médio do erro (RQME) e índice de concordância de Willmott (I)

\begin{tabular}{cccc}
\hline Método $^{\mathbf{1}}$ & $\overline{\mathbf{U}}_{\mathbf{z}}\left(\mathbf{m ~ s}^{\mathbf{- 1}}\right)$ & $\mathbf{R Q M E}\left(\mathbf{m ~ s}^{\mathbf{- 1}}\right)$ & $\mathbf{I}$ \\
\hline $\mathrm{OBS}$ & $3,32( \pm 1,06)$ & & \\
$\mathrm{CO}$ & $3,36( \pm 1,18)$ & 0,24 & 0,9885 \\
$\mathrm{CT}_{\mathrm{RP}}$ & $3,41( \pm 1,20)$ & 0,26 & 0,9864 \\
$\mathrm{CT}_{\mathrm{MD}}$ & $3,38( \pm 1,19)$ & 0,24 & 0,9879 \\
$\mathrm{CT}_{\mathrm{LT}}$ & $3,83( \pm 1,34)^{*}$ & 0,62 & 0,9334 \\
$\mathrm{TA}$ & $3,32( \pm 1,17)$ & 0,23 & 0,9894 \\
$\mathrm{LO}$ & $3,29( \pm 1,15)$ & 0,22 & 0,9896 \\
$\mathrm{RP}$ & $3,37( \pm 1,19)$ & 0,24 & 0,9881 \\
$\mathrm{MD}$ & $3,37( \pm 1,19)$ & 0,24 & 0,9880 \\
\hline
\end{tabular}

* A média dos valores estimados difere estatisticamente da média dos observados pelo teste-t $(\mathrm{p}<0,05)$

(1) Valor observado (OBS); método convencional (CO); convencional-Thom em função de $\lambda$ estimado pela relação de Raupach (1992) $\left(\mathrm{CT}_{\mathrm{RP}}\right)$; com $\lambda$ determinado pela relação de MacDonald et al. (1998) $\left(\mathrm{CT}_{\mathrm{MD}}\right)$ e com $\lambda$ médio da literatura $\left(\mathrm{CT}_{\mathrm{LT}}\right)$; método de Takagi (TA); Lloyd (LO); Raupach (RP) e MacDonald (MD) 


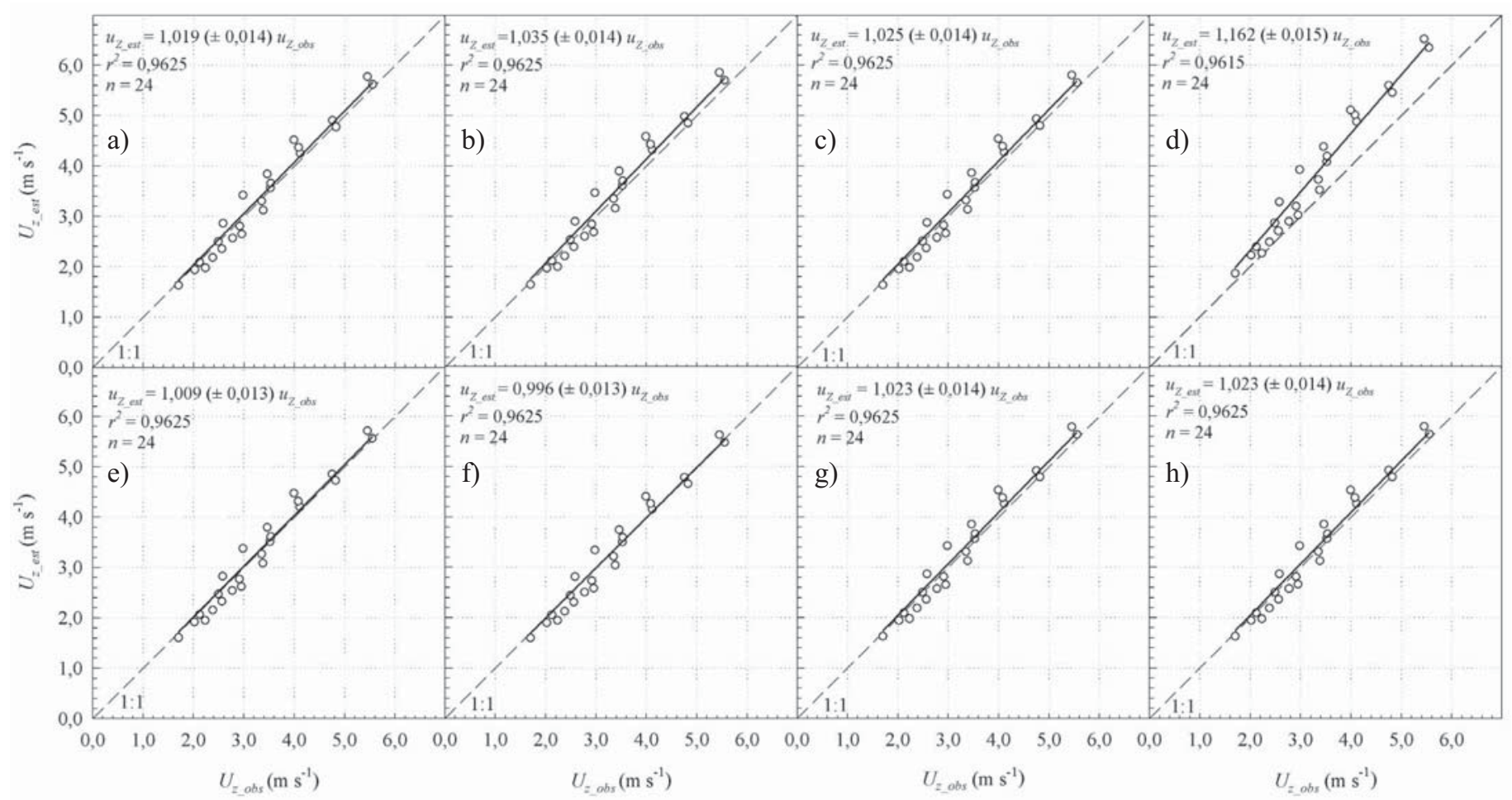

Figura 2 - Regressão linear entre a velocidade do vento observada $\left(U_{z_{\text {_obs }}}\right)$ e a estimada pelo perfil logarítmico do vento $\left(U_{z_{-} \text {est }}\right)$ para cada nível de medida em função de $\mathrm{d}$ e $\mathrm{z}_{0}$ determinado pelo método convencional (a), convencional-Thom utilizando a densidade de rugosidade $(\lambda)$ estimada pela relação de Raupach (1992) (b), com $\lambda$ determinado pela relação de MacDonald et al. (1998) (c), $\lambda$ médio da literatura (d), pelo método de Takagi (e), Lloyd (f), Raupach (g) e MacDonald (h). Valores entre parênteses representam o erro padrão de estimativa do coeficiente angular. Coeficiente de determinação da regressão $\left(\mathrm{r}^{2}\right)$ e número de amostras utilizadas $(\mathrm{n})$.

de forma satisfatória a rugosidade da superfície subjacente. Os parâmetros de rugosidade não representativos, determinados pelo $\mathrm{CT}_{\mathrm{LT}}$, foram proporcionados pelo baixo valor de $\lambda$ utilizado em sua iteração, aliado a elevada sensibilidade de $\mathrm{CT}$ a variações em $\lambda$. Apesar do valor médio da literatura não ter apresentado resultados satisfatórios para vegetação esparsa, ele deve ser avaliado também para vegetação homogênea $(\Lambda>1)$, visto que, neste caso, $\lambda$ mostra-se constante, e que as relações analíticas baseadas nos modelo de Raupach e MacDonald não se aplicam.

A velocidade do vento apresentou dependência inversamente proporcional a d e a $\mathrm{z}_{0}$. Porém, observou-se sensibilidade superior das estimativas de $\mathrm{U}_{\mathrm{z}}$ a mudanças em $\mathrm{z}_{0}$ com relação à observada para d. Na média do perfil, incrementos de $10 \%$ em d implicaram em diminuição de $\mathrm{U}_{\mathrm{z}}$ ao redor de $1,3 \%$, enquanto para $z_{0}$ essa variação resultou em diferenças de $-3,4 \%$. A diferença $(47,2 \%)$ observada entre $d$ determinado pelo método que apresentou estimativas de $\mathrm{U}_{\mathrm{z}}$ com concordância superior (LO) e o de maior divergência $\left(\mathrm{CT}_{\mathrm{LT}}\right)$ resultou em variações de $-6,2 \%$ em $\mathrm{U}_{\mathrm{z}}$, enquanto a diferença entre $\mathrm{z}_{0}$ desses métodos $(-46,8 \%)$ incrementou $\mathrm{U}_{\mathrm{z}}$ em $16,1 \%$. Isto indicou que os valores de $\mathrm{z}_{0}$ de $\mathrm{CT}_{\mathrm{LT}}$ inferior ao dos outros métodos foi o responsável pela superestimativa de $\mathrm{U}_{\mathrm{z}}$.

\section{CONCLUSÕES}

Baseado nos resultados obtidos para os parâmetros de rugosidade sobre vegetação esparsa e de porte alto em ambiente semi-árido do Sahel, pode-se concluir que:

- a abordagem convencional proporciona estimativas aceitáveis do deslocamento do plano zero (d) e do comprimento de rugosidade $\left(\mathrm{z}_{0}\right)$;

- os valores de $\mathrm{d}$ e $\mathrm{z}_{0}$ determinados em função da velocidade de fricção estimada por correlação de vórtices turbulentos na solução da lei logarítmica descrevem melhor a rugosidade da superfície do que aqueles determinados pelos demais métodos que utilizam a lei logarítmica ou os baseados na estrutura física da vegetação;

- as relações entre as características estruturais da vegetação e o transporte de momentum descrevem apropriadamente a rugosidade da superfície;

- no método convencional-Thom, o valor de $\lambda$ calculado pelas relações analíticas de MacDonald e Raupach produzem resultados satisfatórios, o que não é observado quando se utiliza o valor médio da literatura. Contudo deve-se avaliar o método para superfície homogênea, considerando $\lambda$ da literatura; 
- os valores dos parâmetros de rugosidade que descrevem melhor à vegetação são $\mathrm{d}=0,95 \mathrm{~m}$ e $\mathrm{z}_{0}=0,204 \mathrm{~m}$, determinados pelo método de Lloyd. O valor de d representa $46 \%$ da altura média da vegetação e $\mathrm{z}_{0} 10 \%$, sendo $\lambda=0,185$;

- a velocidade horizontal do vento apresenta baixa sensibilidade aos parâmetros de rugosidade, porém é mais sensível a $\mathrm{z}_{0}$ do que a $\mathrm{d}$.

\section{AGRADECIMENTOS}

Os autores agradecem ao Departamento de Meteorologia da Universidade de Reading, UK, pela coleta dos dados de correlação de vórtices turbulentos e de perfil de vento. Financiada pela UK Terrestrial Initiative in Global Environmental Research. Ao Instituto de Manejo Ecológico da Universidade de Edinburgh, UK, pelas análises da estrutura física da vegetação. E a equipe do sistema de informação do HAPEX-Sahel por disponibilizar os dados.

\section{REFERÊNCIAS BIBLIOGRÁFICAS}

DE BRUIN, H. A. R.; MOORE, C. J. Zero-plane displacement and roughness length for tall vegetation, derived from a simple mass conservation hypothesis. Boundary-Layer Meteorology, v. 31, n. 1, p. 39-49, 1985.

DE BRUIN, H. A. R.; VERHOEF, A. A new method to determine the zero-plane displacement. Boundary-Layer Meteorology, v. 82, n. 1, p. 159-164, 1997.

DOLMAN, A. J.; LLOYD, C. R.; CULF, A. D. Aerodynamic roughness of an area of natural open forest in the Sahel. Annales Geophysicae, v. 10, n. 11/12, p. 930-934, 1992.

GARRATT, J. R. Surface influence upon vertical profiles in the atmospheric near-surface layer. Quarterly Journal of the Royal Meteorological Society, v. 106, n. 450, p. 803$819,1980$.

JACOBS, A. F.; VAN BOXEL, J. H. Changes of the displacement height and roughness length of maize during a growing season. Agricultural and Forest Meteorology, v. 42, n. 1, p. 53-62, 1988.

LETTAU, H. Note on aerodynamic roughness-parameter estimation on the basis of roughness-element description. Journal of Applied Meteorology, v. 8, n. 5, p. 828-832, 1969.
LEVY, P. E.; JARVIS, P. G. Direct and indirect measurements of LAI in millet and fallow vegetation in HAPEX-Sahel. Agricultural and Forest Meteorology, v. 97, n. 3, p. 199$212,1999$.

LLOYD, C. R.; GASH, J. H. C.; SIVAKUMAR, M. V. K. Derivation of the aerodynamic roughness parameters for a Sahelian savannah site using the eddy-correlation technique. BoundaryLayer Meteorology, v. 58, n. 3, p. 261-271, 1992.

LLOYD, C. R. et al. A comparison of surface flux at the HAPEX-Sahel fallow bush sites. Journal of Hydrology, v. 188/189, n. 1, p. 400-425, 1997.

LYRA, G. B. Parâmetros de rugosidade aerodinâmica sobre vegetação esparsa. 2005. 75 f. Tese (Doutorado em Agronomia) - Escola Superior de Agricultura "Luiz de Queiroz”, Universidade de São Paulo, Piracicaba, 2005.

MacDONALD, R.W.; GRIFFITHS, R. F.; HALL, D. J. An improved method for the estimation of surface roughness of obstacle arrays. Atmospheric environment, v. 32, n. 11, p. 1857-1864, 1998.

MOLION, L. C. B; MOORE, C. J. Estimating the zero-plane displacement for tall vegetation using a mass conservation method. Boundary-Layer Meteorology, v. 26, n. 2, p. 115-125, 1983.

MONIN, A. S.; OBUKHOV, A. M. Basic relationships of turbulent mixing in the surface layer of the atmosphere. Doklady Akademii Nauk SSSR, v. 151, n. 24, p. 163-187, 1954.

MONTEITH, J. L.; UNSWORTH, M. H. Principles of environmental physics. 2th ed. New York: Edward Arnold, 1990. $291 \mathrm{p}$.

MUNRO, D. S.; OKE, T. R. Estimating wind profile parameters for tall dense crops. Agricultural Meteorology, v. 11, n. 2, p. 223-228, 1973.

PEREIRA, A.R. Introdução à micrometeorologia. Piracicaba: ESALQ, Departamento de Ciências Exatas, 2002. 74p.

RAUPACH, M. R.; THOM, A. S.; EDWARDS, I. A wind tunnel study of turbulent flow close to regularly arrayed rough surfaces. Boundary-Layer Meteorology, v. 18, n. 4, p. 373-397, 1980. 
RAUPACH, M. R. Drag and drag partition on rough surfaces. Boundary-Layer Meteorology, v. 60, n. 4, p. 375-395, 1992.

RAUPACH, M. R. Simplified expressions for vegetation roughness length and zero-plane displacement as functions of canopy height and area index. Boundary-Layer Meteorology, v. 71, n. 1, p. 211216, 1994.

RAUPACH, M. R. Corrigenda for Raupach (1992) and Raupach (1994). Boundary-Layer Meteorology, v. 76, n. 3, p. 303304, 1995.

RIGH, E. Z. Balanço de energia e evapotranspiração de cafezal adensado em crescimento sob irrigação localizada. 2004. 151 f. Tese (Doutorado em Agronomia) - Escola Superior de Agricultura "Luiz de Queiroz", Universidade de São Paulo, Piracicaba, 2004.

SCHAUDT, K. J. A new method for estimating roughness parameters and evaluating the quality of observations. Journal of Applied Meteorology, v. 37, n. 5, p. 470-476, 1998.

SHAW, R. H.; PEREIRA, A. R. Aerodynamic roughness of a plant canopy: a numerical experiment. Agricultural Meteorology, v. 26, n. 1, p. 51-65, 1982.
ShUtTLEWORTH, W. J. etal. An integrated micrometeorological system for evaporation measurement. Agricultural and Forest Meteorology, v. 43, n. 3-4, p. 295-317, 1988.

TAKAGI, K. et al. An alternative approach to determining zero-plane displacement, and its application to a lotus paddy field. Agricultural and Forest Meteorology, v. 115, n. 3/4, p. 173-181, 2003.

THOM, A. S. Momentum absorption by vegetation. Quarterly Journal of the Royal Meteorological Society, v. 97, n. 414, p. 414-428, 1971.

WALLACE et al. Hapex-Sahel southern super-site report: an overview of the site and the experimental programme during the intensive observation period in 1992. Wallingford: Institute of Hydrology, 1994. 55p.

WIERINGA, J. A. revaluation of the Kansas mast influence on measurements of stress and cup anemometer overspeeding. Boundary-Layer Meteorology, v. 18, n. 4, p. 411-430, 1980.

WIERINGA, J. A. Representative roughness parameter for homogeneous terrain. Boundary-Layer Meteorology, v. 63, n. 4, p. 323-363, 1993.

WILlMOTT, C. J. On the validation of models. Physical Geography, v. 2, n. 2, p. 184-194, 1981. 